\title{
واقع التخطيط الاستراتيجي بكليات جامعة مصراتة- كلية التربية نموذجاً
}

$$
\begin{aligned}
& \text { أ. زهرة الشريف عبدالسلام } \quad \text { أ. غادة جمال بن إسماعيل } \\
& \text { كلية التربية- جامعة مصراتة كلية التربية- جامعة مصراتة }
\end{aligned}
$$

الملخص:

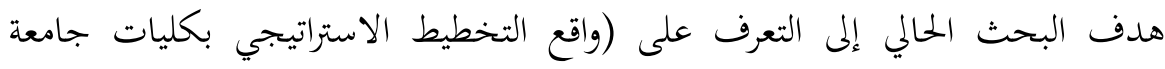
مصراته- كلية التربية نموذجاً) ولتحقيق هذا التهرف على الهدف تم طرح التساؤل الرئيسي الآتي:

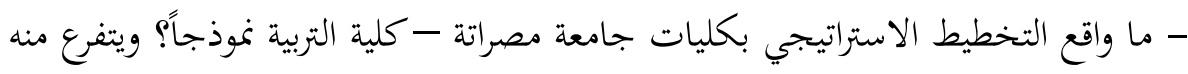

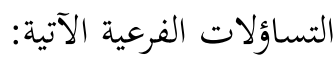

1- 1 ما مستوى انتشار ثقافة التخطيط الاستراتيجي في الكلية ؟

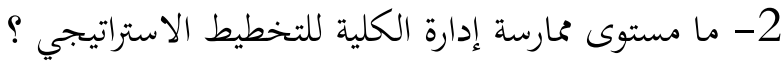

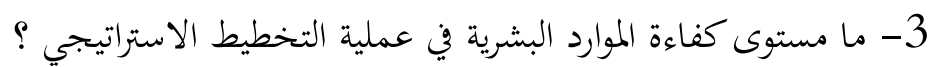

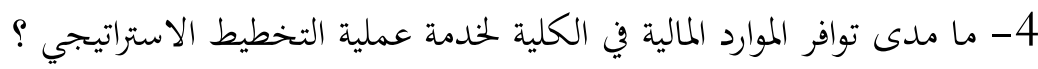
5- هل توجد فروق ذات دلالة إحصائية في استجابات أفراد العينة لواقع التخطيط

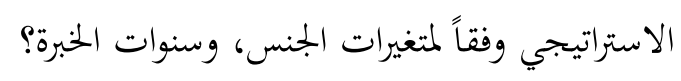

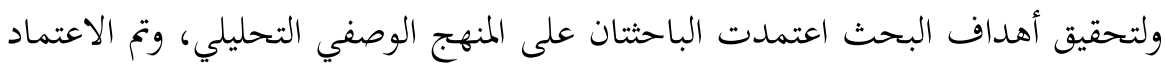

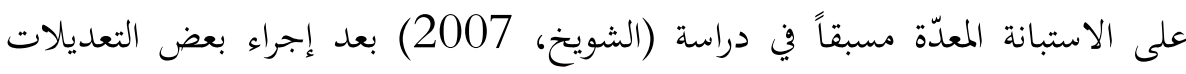

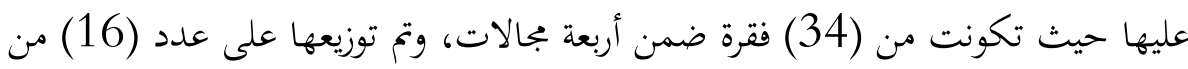

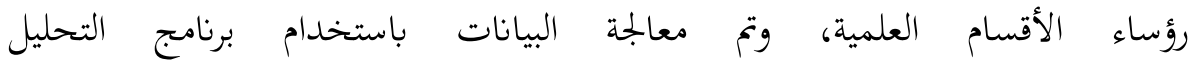
الإحصائي"SPSS".وقد توصل البحث إلى النتائج الآتية:

${ }^{1}$ zahra.ashareef2000@gmail.com 


$$
\begin{aligned}
& 1 \text { - أن مستوى انتشار ثقافة التخطيط الاستراتيجي لدى أفراد العينة جاء بنسبه } \\
& \text { متوسطة. } \\
& \text { 2- أن مستوى محارسة التخطيط الاستراتيجي لدى أفراد العينة جاء بنسبه متوسطة. } \\
& \text { 3- أن مستوى كفاءة الموارد البشرية لدى أفراد العينة جاء بنسبه متوسطة. } \\
& \text { 4- أن توافر الموارد المالية في الكلية لخدمة عملية التخطيط الاستراتيجي جاءت بنسبة } \\
& \text { منخفضة. } \\
& \text { 5- عدم وجود فروق ذات دلالة إحصائية في استجابات أفراد العينة حول واقع } \\
& \text { التخطيط الاستراتيجي وفقاً لمتغيرات الجنس، سنوات الخبرة. } \\
& \text { الكلمات المفتاحية: التخطيط - التخطيط الاستراتيجي - جامعة مصراتة }
\end{aligned}
$$

\section{Abstract}

The reality of strategic planning of the colleges of Misurata University - college of education as amodel.

The goal of current research to identify the: what is the reality of strategic planning of the colleges of Misurata University - college of education as amodel.

To achieve this objective, the main question was put as follows:The following subsidiary questions wrapping it:

1 - what is the level of the spread of aculture of strategic planning in the college?

2- what is the standard practice of the college strategic planning?

3- what is the level of the efficiency of human resources in strategic planning?

4- what is the availability of financial resources in the college to serve the strategic planning process?

5- IS there a statistically significant differences in the responses of the sample members of the reality of strategic planning in accordance with the veriables of sex and years of experience?

In order to achieve the objectives of the research on the descriptive approach adopted the researchers analytical work, and rely on the resolution prepared in advance in the study(Shuwaikh, 2007) after 
some modifications, consisting of (34) paragraph within the four areas.

It was distributed to a number (16) of the heads of the scientific departments, and processing of the data using the statistical analysis program(SPSS)

The research reached the following results:

1- That the level of the spread of a culture of strategic planning to the sample members cam by medium.

2- That the Level of the exercise of the college strategic planning came by an average of.

3- The level of the efficiency of the human resources of the members of the sample was increased by an average of.

4- The availability of financial resources in the college to serve the strategic planning process at low.

5- The absence of statistically significant differences in the responses of the members of the sample on the reality of strategic planning in accordance with the veriables of sex and years of experience.

Keywords: Planning- Strategic Planning-Misurata University

1

يعتبر التخطيط من أهم العمليات الأساسية للإدارة، ومن الصعب تصور منظمة ما تمارس نشاطها بدون هذه العملية، فمن خلاله تتحدد الغايات والأهداف الأساسية والإجراءات

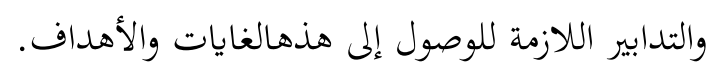

والتخطيط الاستراتيجي كأحد أنواع التخطيط إنما يعبر عن عملية تخطيط تستند إلى فهم واقعي وعميق لما يدور في بيئةالمنظمة الداخلية، ومحاولة التعرف على نقاط القوة ونقاط

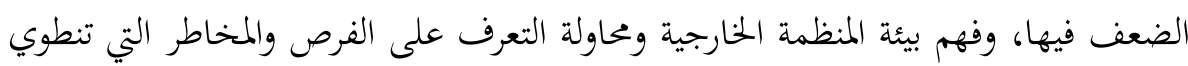

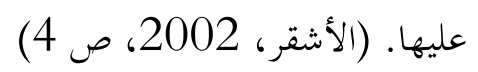

يعد التخطيط الاستراتيجي من المفاهيم الإدارية الحديثة التي ظهرت في منتصف القرن التاسع عشر، وارتبط هذا المفهوم بالمفاهيم العسكرية ثم الصناعية. حيث إن مفهوم التخطيط الاستراتيجي يترابط بمفهوم الإستراتيجية، فهو الأسلوب الذي تختاره الإدارة 
للاستفادة من الموارد المتاحة لها ولتحقيق أفضل النتائج. (السلمي، 2000، ص 120) وعلى الرغم من أن تبني التخطيط الاستراتيجي في المؤسسات التعليمية بدأ متأخراً عن مجال

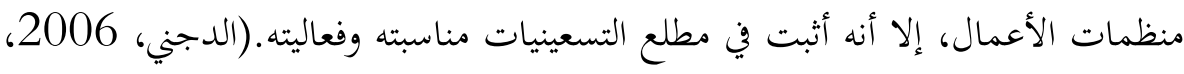

ويعتبر التعليم العالي من أهم المراحل التعليمية، لأفها تعد استكمالاً لما تم تحقيقه في مراحل التعليم الأساسي والثانوي، ولذلك فإن تحقيق الأهداف التربوية التي يتوخاها المجتمع يعتمد على قدرة النظام التربوي على تحقيق أهدافه في هذه المراحل. (الدجني، 2006، (4) إن الفهم السليم لأهمية التخطيط الاستراتيجي من صانعي القرار في المؤسسات التعليمية

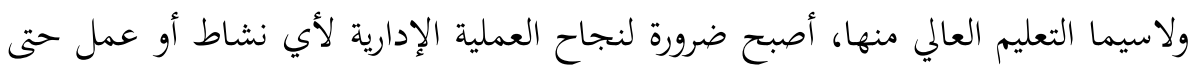
تبقى هذه المؤسسات صامدة أمام التغييرات المستمرة بأسلوب علمي سليم. وقد أوضحت (الشيخي، 2008) أن نتائج الدراسات والتقارير التي أجريت في كثير من الدول أشارت إلى أن أغلبية المديرين لا يملكون رؤية واضحة للمفهوم العلمي للتخطيط الاستراتيجي بعنناه المعاصر والدقيق. (الشيخي، 2008، ص 6) وهذا ما تحاول الدراسة

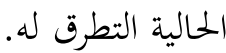

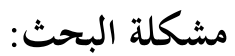
في ظل بيئة تتميز بعدم الاستقرار وعدم الثبات، وتسارع وتيرة التغيرات على كافة الأصعدة الاقتصادية والسياسية والاجتماعية والثقافية، يكون من الضروري فهم المسؤولين على عملية صناعة واتخاذ القرار للتخطيط الاستراتيجي وممارسته بشكل فعّال، من أجل زيادة فرص بقاء ونجاح المؤسسات لاسيما التعليمية منها التي يديروغا، ولضمان قيام هذه 
المؤسسات بالأدوار المناطة بها على أكمل وجه. ومن هنا جاء هذا البحث للتعرف على واقع التخطيط الاستراتيجي بكليات جامعة مصراتة. ومما سبق يمكن تحديد مشكلة البحث

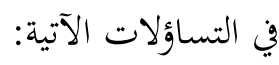
- ما واقع التخطيط الاستراتيجي بكليات جامعة مصراتة - كلية التربية نموذجاً؟ ويتفرع منه التساؤلات الفرعية الآتية: 1- ما مستوى انتشار ثقافة التخطيط الاستراتيجي في الكلية ؟ التهاته

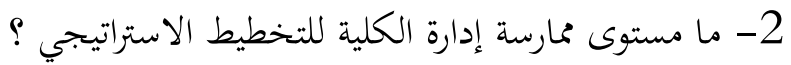

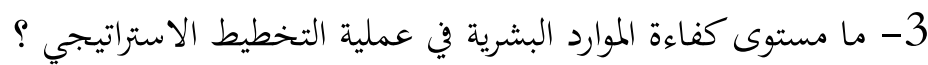
4- ما مدى توافر الموارد المالية في الكلية لخدمة عملية التخطيط الاستراتيجي ؟ 5- هل توجد فروق ذات دلالة إحصائية في استجابات أفراد العينة لواقع التخطيط الاستراتيجي وفقاً لمتغيرات الجنس، وسنوات الخبرة؟ حدود البحث: : يحدّ البحث الحالي الحدود الآتية: 1- الحدود الموضوعية: يقتصر البحث على التعرف على واقع التخطيط الاستراتيجي بكليات جامعة مصراتة. 2- الحدود المكانية: أجري البحث بكلية التربية. 3- الحدود الزمانية: أجري البحث خلال العام الجامعي 2019. 4- الحدود البشرية: أجري البحث على جميع رؤساء الأقسام العلمية بكلية التربية. أهداف البحث: يسعى هذا البحث إلى تحقيق الأهداف الآتية: 1- التعرف على مستوى انتشار ثقافة التخطيط الاستراتيجي في الكلية . 2- التعرف على مستوى ممارسة إدارة الكلية للتخطيط الاستراتيجي. 3- التعرف على مستوى كفاءة الموارد البشرية في عملية التخطيط الاستراتيجي. 
4- التعرف على مدى توافر الموارد المالية في الكلية لخدمة عملية التخطيط الاستراتيجي. 5- التعرف على ما إذا هناك فروق ذات دلالة إحصائية في استجابات أفراد العينة لواقع التخطيط الاستراتيجي وفقاً لمتغيرات الجنس، وسنوات إنسات الحبرة. أهمية البحث:

1- يستمد البحث الحالي أهميته من أهمية التخطيط الاستراتيجي حيث يعتبر عنصراً مهماً من عناصر استقرار المؤسسات، والمحافظة على توازها واستمرارها. 2-إثراء معرفة رؤساء الأقسام بمفهوم التخطيط الاستراتيجي، الأمر الذي قد يسهم في رفع مستوى أدائهم الإداري في هذا الجانب.

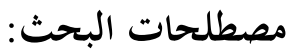

1-التخطيط: يعرّف بأنه عملية منظمة واعية لاختيار أحسن الحلول الممكنة للوصول إلى أهداف معينة. (حافظ، البحيري، 2006، ص 15) 2- التخطيط الاستراتيجي: ويعرّف بأنه : العملية التي يتحدد من خلالها الغايات المؤسسية بعيدة المدى، وانتقاء الوسائل أي السياسات والإستراتيجيات وتخصيص الموارد وتطوير الخطط طويلة المدى لبلوغ الغايات.(يونس، 2009، ص 75) وتبنت الباحثنين تعريف (غنيم،2001) القائل بأن التخطيط الاستراتيجي هو" عملية اتخاذ القرارات ووضع أهداف وإستراتيجيات وبرامج زمنية مستقبلية وتنفيذها ومتابعتها" . (غنيم، 2001، ص 238) 3- جامعة مصراتة: هي إحدى الجامعات الليبية مقرها مدينة مصراتة، وتضم ستة عشر كلية بين العلوم الإنسانية والتطبيقية. (https.//ar.m.wikpedia.org) 


\section{الإطار النظري ودراسات سابقة:

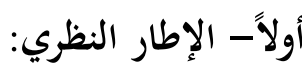

1- مفهوم التخطيط الاستراتيجي: يعد التخطيط الاستراتيجي أسلوبا جديداً في التخطيط التربوي والإدارة التعليمية، والهدف منه في مجال التعليم يتمثل في تعزيز عملية التكيف، والانسجام بين المؤسسة التعليمية، والبيئية التي يغلب عليها طابع التغيير، وذلك

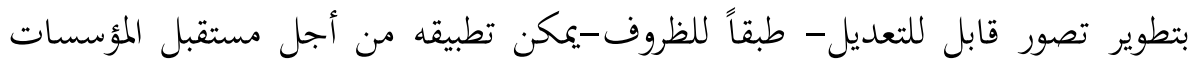

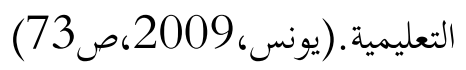
حيث عرّف( درويش وتكلا) التخطيط الاستراتيجي بأنه" أسلوب علمي عملي للربط بين الأهداف والوسائل المستخدمة لتحقيها ورسم معالم الطريق الذي يمدد القرارات والسياسات، وكيفية تنفيذها مع محاولة التحكم في الإحداث بإتباع سياسات مدروسة محددة الأهداف والنتائج" .(درويش،تكلا، 1980، ص صديع 237) ويعرّفه (غطاس، د ت) نقلاً عن (نورالدين، 2008) بأنه: "عملية اتخاذ القرارات فيما يتعلق بأهداف المؤسسة، واستخدام الموارد وتطبيق السياسات من أجل تحقيق هذه

$$
\text { الأهداف". (نور الدين، 2008، ص61) }
$$

ويترابط مفهوم التخطيط الاستراتيجي مع مفهوم الإستراتيجية، حيث تعرف الإستراتيجية في قاموس (Webster's New World Dictionary) بأهما " علم تخطيط وتوجيه العمليات الحربية " أذن يمثل التخطيط أهم مكون في هذا التعريف

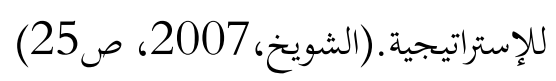
وتعرّف الإستراتيجية بأها "بموعة الخطط الموجهة التي تساعد الإدارة على تحقيق المسار الذي اختارته والاستفادة من الفرص المحيطة بها، ومواجهة القيود والتهديدات والمخاطر التي لئي

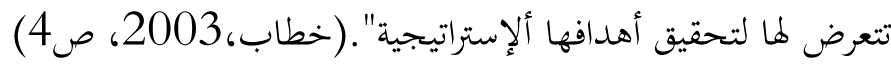


2- مفاهيم أساسية مرتبطة بالتخطيط الاستراتيجي:

أ- التفكير الاستراتيجي: يعرّف التفكير بأنه سلسلة من العمليات المعقدة التي بترى في الدماغ البشري بسرعة مذهلة، مهمتها تبسيط الأمور التي تشغل الذهن، وتحليلها إلى عناصر أولية قابلة للربط والمقارنة والعرض ومن تمخ الخروج بتصور أو نظرية تشكل قاعدة ثابتة للتطبيق العملي، والتفكير الحر يشكل عائقا في وجه التخطيط لأنه يرهق الذهن بكثرة المعلومات التي لا لزوم لها في موضوع يجري التخطيط له بشكل محدد، ومن هنا نشأ ما يمسي بالتفكير الاستراتيجي والذي هو مسار فكري محدد له خط سير واضح به وله

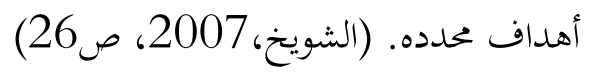

وتبنت الباحثتان الرأي الذي قال بأن التفكير الاستراتيجي خطوة أولى ومهمة من خطوات التخطيط الاستراتيجي ويرتبط التفكير ارتباطا وثيقا كصفة لازمه بالقادة والمخططين الاستراتيجيين؛ لأن أول خطوة في التخطيط الاستراتيجي هو القيام بتشخيص وتحليل للظروف المحيطة، ومن ثم وضع رؤية وبدائل وكل ذلك لا يأتي إلا بالتفكير الاستراتيجي.

ب- التخطيط طويل المدى: للتمييز بين كل من التخطيط الاستراتيجي والتخطيط

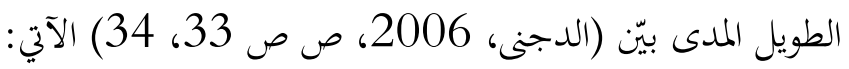
1- التخطيط طويل المدى يفترض أن الاتجاهات المستقبلية هي عبارة عن امتداد للوضع السابق، كما أن الإدارة العليا تفترض بأن الاداء المستقبلي سيكون أفضل من الأداء في الماضي، ولذلك غالبا ماتضع أهدافاً متفائله. أما في التخطيط الاستراتيجي فلا يفرض أن يكون المستقبل دائما أفضل من الماضي أو امتدات له، وعليه فالخطوة الأولى هي تحليل وتحديد الاتحاهات والفرص التى تساعد في تغيير الاتحاهات السابقه وإحداث تطورات في أداء المؤسسة المستقبلي، وهذا يتطلب تغييرات أساسية في الأهداف والاستراتيجيات والأساليب. 
2- يعتمد التخطيط الطويل المدى على حقائق في شكل أرقام وبيانات محددة بزمن محدد، فن حين يعتمد التخطيط الاستراتيجي على قيم تعكس إيمان الإدارة بأهداف تضع إمكانيتها وجهودها للتمسك بها.

3- يركز التخطيط طويل المدى علي مرحلة الإعداد والتنفيذ، بينما يكون التركيز في التخطيط الاستراتيجي علي مرحلة التفكير والتكوين.

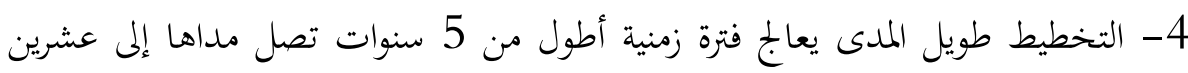

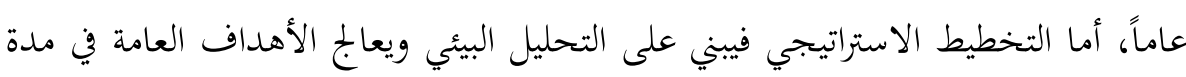

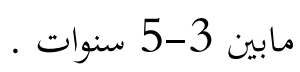

5- في التخطيط الطويل المدى تكون إجراءات ملزمة غير مرنة ولا يمكن بتحاوزها، بينما في

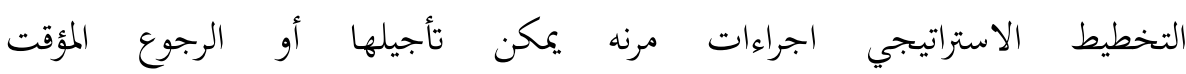

$$
\text { عنها.(الأشقر، 2002، ص252) }
$$

كما أشار "الدجني" على أن هناك ترابط بين أنواع التخطيط بصفه عامه والتخطيط

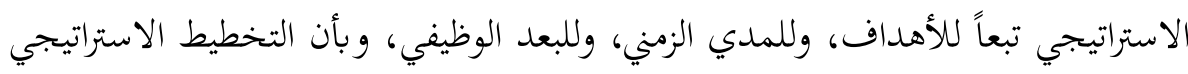

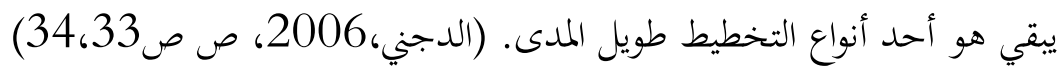
ج- أهمية التخطيط الاستراتيجي وفوائده: يمكن توضيح أهمية التخطيط الاستراتيجي في

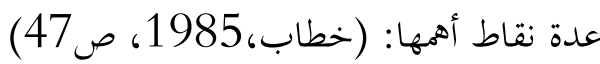
1- تحقيق التنسيق بين مختلف أوجه النشاط والالتزام بالأهداف الإستراتيجية التي تضعها الإدارة. 2- زيادة قدرة الإدارة على السيطرة على الموارد المتاحة، وترشيد تخصيصها في حدود القيود المفروضة عليها. 3- توفير أطار مرشد للإدارة في اتخاذ القرارات، وتحقيق القدرة على التأثير والقيادة. وأما عن فوائد التخطيط الإستراتيجي فأنه يحقق العديد من الفوائد أهمها: 1- توحيد جميع الجهود الإدارات داخل المؤسسة نحو غايات واحدة. 
2- تحديد الأهداف والتوجه اللازم لمستقبل المؤسسة ككل. 3- جعل المديرين باستمرار أكثر استجابةً ووعياً بظروف البيئة سواء داخلية أم خارجية للمؤسسة وتغيراتا. 4- تنمية عادات التفكير في المستقبل، ممايوفر للمؤسسة إمكانية أفضل لرؤية المستقبل من خلال تحديد نقاط القوة وضعف المؤسسة. 5- توفير فرصة مشاركة جميع المستويات الإدارية في عملية التخطيط.(الشويخ، 2007، وصعفة

\section{د- خصائص التخطيط الاستراتيجي}

ترى (حريري، 2007) نقلاً عن( يونس ،2009) أنسريجيط التخطيط الاستراتيجي يتسم بما يأتي:

1- الشمول والتكامل: أيتعدي حدود الأشياء التي يمكن التحكم فيها ليتعرف عل المتغيرات البيئة المحيطة كالسياسية والاقتصادية والاجتماعية والتكنولوجية كي تتؤخذ فلا الاعتبار عند اتخاذ القرار.

2- التفاعل بين مستويات التخطيط وتقديم التغذية الراجعة والتفاعل المستمر بين كل المعنيين سواء مخططين أومنفذين. 3- المرونة وهي سمة أساسية بسبب الطابع المتغير للبيئة التعليمية ذلك لوجود تطورات عملية وتقنية وصناعية واتصالية. 4- التفاعل بين التخطيط والتنفيذ: فالتخطيط يعتمد على سلامة التنفيذ.

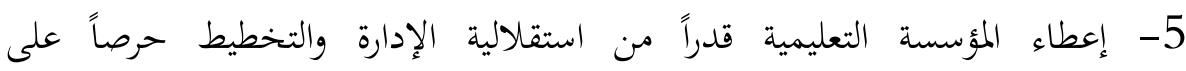

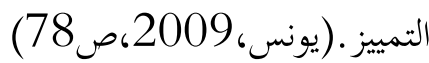
هـ- مبررات التخطيط الاستراتيجي: وتتلخص أهم مبررات التخطيط الاستراتيجي في

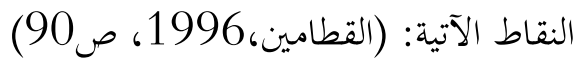


1- أكتشاف أخطاء في عمل المؤسسة وسبب الاخفاق في الوصول الى نتائج متطابقة في الاهداف الموضوعه.

2- وجود فجوة ملفته للنظر فن أداء المؤسسة وذلك عندما تصبح نتائج الأداء تبعد كثيراً عن التوقعات، ويحدث ذلك عندما تختلف المؤشرات الأدائية بصورة ملفته للنظر عن مثيلاتما عند المؤسسات السائدة، وفي حالة حدوث ذلك تطرح كل الأمور والمسلمات السائدة لدى المؤسسة على طاولة البحث وتتجه الأنظار المتشكلة إلى رسالة المؤسسة والأهداف الاستراتيجية وخططها أو سياستها عن السبب أو الأسباب، وذلك مما يطرح عملية التخطيط من جديد. 3- تولي مدير عام جديد لمهامة في المؤسسة: إن المعتاد في عالم الإدارة أن لكل مدير عام

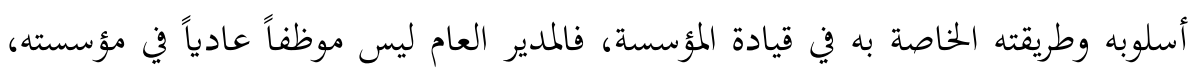
فهو يتمتع بسلطات ومسؤوليات واسعة تتيح له تغيير معالم العمل المؤسسي للوجهة التي يراها أكثر ملائمة لانجاز أهداف مؤسسته، فقد يري أن الوضع الراهن لايتفق مع مايجب لئب

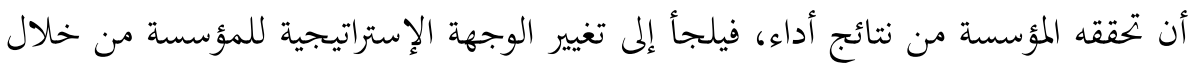
البدء بتغيير رسالتها أو الأهداف الإستراتيجية القائمة أو الخطط والسياسات ادلهات المتبعة.

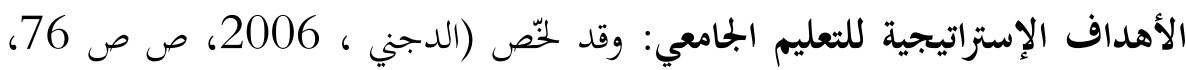
77) الأهداف الاستراتيجية للتعليم الجامعي كالآتي:

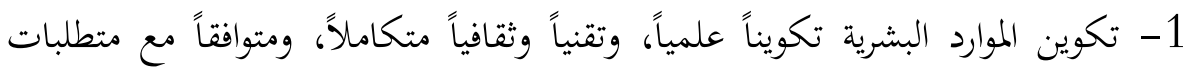
العصر ومتغيراته، وتوفير سبل التنمية المستمرة لتلك الموارد بما يهيئها للمشاركة الفاعلة المتميزة في تفعيل ثروات المجتمع وتحقيق نموه وتطوره ودعم قدراته.

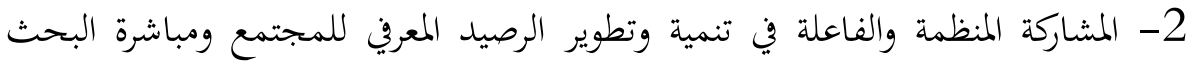
العلمي المنظم لحل مشكلات المجتمع والمساهمة في التنمية القومية، وذلك من خلال التوظيف المخطط والتنمية المستمرة للقدرات والموارد العلمية التي تسهم في زيادة قدراته 
3- استثمار العلم والتقنية في إدارة مؤسسات التعليم الجامعي والعالي، وتنمية علاقات التعاون والتفاعل الديمقراطي بين عناصرها . 4- المساهمة الايجابية في دراسة وحل مشكلات البمتمع، وتوفير المعرفة والثقافة، والعمل على نشرها، والمشاركة في التوعية بالمحافظة على البيئة. 5- تطوير منظومة التعليم الجامعي والعالي للتوافق مع معايير والنظم العالمية. متطلبات التخطيط الاستراتيجي في مؤسسات التعليم العالي: أوردها (الدجني،

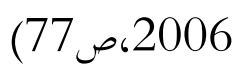
1- هيكل تنظيمي واضح ومناسب للمؤسسة، أيضاً إدارة وموظفون ملتزمون بالخطة. 2- توفر الإمكانات المادية والمهارات المناسبة والمتنوعة للقيام بالتخطيط. 3- توفر صورة واظحه عن بيئة الجامعة وإدراك القصور جيدا. 4- قناعة كاملة للمشاركين بالخطة بحجم الفوائد المترتبة علي تطبيق الخطة الإستراتيجية.

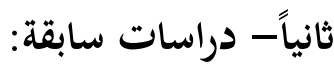

-دراسة نجاة أحممد أبوشناق (2011) (التخطيط الاستراتيجي للتدريب وأثره على أداء العاملين): هدفت الدراسة إلى التعرف على واقع التخطيط الاستراتيجي للتدريب في المؤسسة الوطنية للنفط والوقوف على أوجه القصور وأبرز المشاكل التي تواجهه عند تطبيق

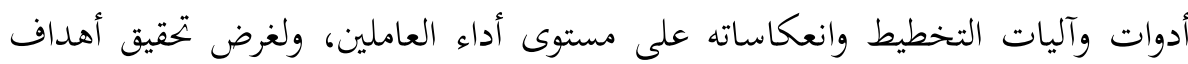
الدراسة قامت الباحثة بتطوير استبانه تكونت من ثلاثة أجزاء الأول يقيس واقع التخطيط الاستراتيجي للتدريب، والثاني يقيس تطبيق استراتيجيات التدريب والثالث مستوي أداء العاملين، وتعتمد منهجية الدراسة على المنهج الوصفي التحليلي، وبلغت عينة البحث "44" مفردة من قيادات المؤسسة. وتوصلت الدراسة لمجموعة من النتائج منها:

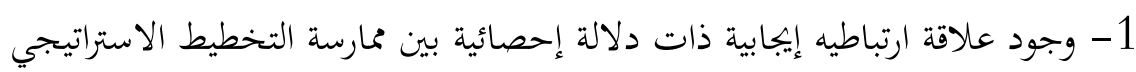
للتدريب ومستوى أداء العاملين. 
2- أثبتت نتائج الدراسة أن للمؤسسة أهدافا من التخطيط الاستراتيجي للتدريب وإبعاده الأساسية تكمن في تحديد الرسالة ، وتطوير الأهداف والاستراتيجيات.

- دراسة عاطف عبد الحميد الشويخ (2007) (واقع التخطيط الاستراتيجي في مؤسسات التعليم التقني في محافظات غزة): هدفت الدراسة إلى التعرف على واقع التخطيط الاستراتيجي في مؤسسات التعليم التقني في محافظات غزة، ولتحقيق أهداف الدراسة، اتبع الباحث المنهج الوصفي التحليلي، وبلغت عينة الدراسة (86) عضواً، وقد توصلت الدراسة لمجموعة من النتائج منها: 1- أظهرت الدراسة على أنه توجد علاقة بين التخطيط الاستراتيجي في مؤسسات التعليم التقني ومدى التزام إدارة الكلية بالتخطيط الاستراتيجي . 2- وجود علاقة بين التخطيط الاستراتيجي في مؤسسات التعليم التقني ومدى انتشار ثقافته بما يخدم عملية التخطيط الاستراتيجي .

3- وجود علاقة بين التخطيط الاستراتيجي في مؤسسات التعليم التقني وكفاءة الموارد البشرية، مما يعزز دور الكفاءات العلمية في عملية التخطيط الاستراتيجي. 4- وجود علاقة بين التخطيط الاستراتيجي في مؤسسات التعليم التقني وتوافر الموارد المالية، والتي من شأها تطوير عملية التخطيط الاستراتيجي.

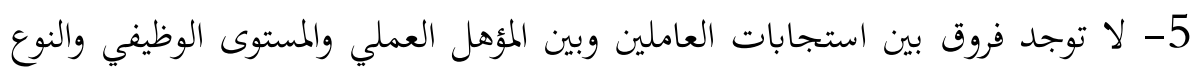
وعملية التخطيط الاستراتيجي، بينما كانت هناك فروق مع اسم الكلية وسنوات الحندمة. 2. إجراءات البحث: وعمبه

أولاًَ- منهج البحث: استخدمت الباحثتان المنهج الوصفي التحليلي الذي يقوم على وصف وجمع وتحليل البيانات تمشياً مع ما هدف إليه البحث. ثانياً- مجتمع البحث: أشتمل مجتمع البحث على جميع رؤساء الأقسام العلمية بكلية التربية البالغ عددهم (17) رئيساً. 
ثالثاً - أداة البحث: اعتمدت الباحثتان على الاستبانة كأداة لجمع البيانات: تم مراجعة الأدبيات التربوية والدراسات السابقة ذات العلاقة بموضوع الدراسة، حيث تم الاعتماد على فقرات الاستبانة في دراسة (الشويخ، 2007). وقد تم وضع مقياس ثلاثي الأبعاد لفقرات

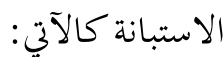

\begin{tabular}{|c|c|c|c|}
\hline غير موافق & محايد & موافق & لتصنيف \\
\hline 1 & 2 & 3 & لدرجة \\
\hline \multicolumn{4}{|c|}{ يقابلها في التحليل } \\
\hline منخفض & متوسط & مرتفع & لتصنيف \\
\hline $1.66-1$ & $2.34-1.67$ & $3-2.35$ & الدرجة \\
\hline
\end{tabular}

- صدق الأداة: تم استخراج صدق الأداة من خلال الصدق الظاهري: حيث عرضت الاستبانة على مجموعة من المحكمين من أعضاء هيئة التدريس بقسم الإدارة والتخطيط التربوي بكلية التربية لإبداء آراءهم حول فقراما وإجراء ما يلزم من تعديلات.

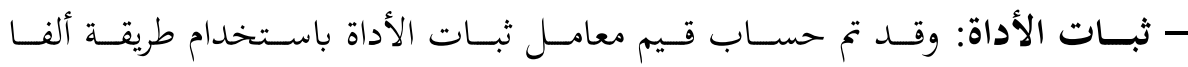
كرونباخ Crompach's Alpha حيث كانت نسبة ثبات الاستبانة 0.95\%.

- تطبيق الأداة: تم تطبيق أداة البحث على جميع أفراد مجتمع البحث البالغ عدده (17) مستجيبا، وأثناء التطبيق فقدت استبانة واحدة، وتم استرجاع عدد(16) استبانة. رابعاً- المعالجة الإحصائية: بعد جمع الاستبانات ثم استخدام حزم التحليل الإحصائية للعلوم الاجتماعية (SPSS) حيث استخدم المتوسطات الحسابية والانحراف المعياري، وكذلك اختبار (تحليل التباين أحادي الإبحاه) لحساب دلالة الفروق الإحصائية لاستجابات عينة البحث وفقاً لمتغيرات الجنس وسنوات الخبرة، كما هو محدد في تساؤلات البحث. 


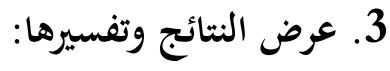

أولا - النتائج التي تتعلق بالتساؤل الأول :

ينص هذا التساؤل على الآتي: ما مستوى انتشار ثقافة التخطيط الاستراتيجي بكليات جامعة مصراتة؟ ولإججابة على هذا التساؤل تم حساب المتوسطات الحسابية والانحراف المعياري لاستجابات أفراد العينة، والجدول رقم (1) يوضح ذلك: جلدول رقم (1) يبين المتوسطات الحسابية والانحراف المعياري لاستجابات أفراد العينة حول المجال الأول

\begin{tabular}{|c|c|c|c|c|}
\hline الرتبة & الانخراف & المتوسط & الفقرات & رقم الفقرة \\
\hline 7 & 0.885 & 1.875 & 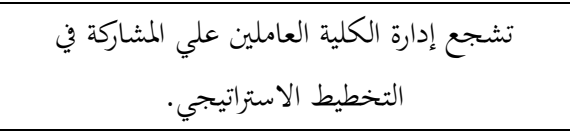 & 1 \\
\hline 3 & 0.834 & 2.187 & تربط إدارة الكلية سياستها الإستراتيجية بثقافة المجتمع & 2 \\
\hline 7 & 0.718 & 1.875 & تحترم إدارة الكلية آراء العاملين فيها حول الخطة & 3 \\
\hline 8 & 0.834 & 1.812 & تراعي إدارة الكلية الثقافة السائدة بين العاملين بما & 4 \\
\hline 6 & 0.853 & 1.937 & 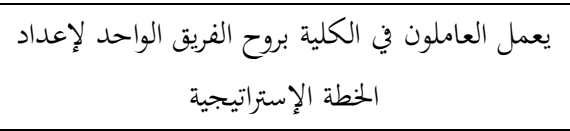 & 5 \\
\hline 6 & 0.928 & 1.937 & 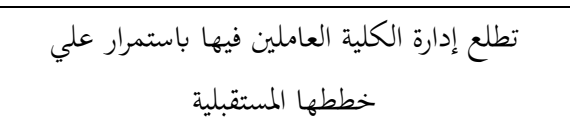 & 6 \\
\hline 5 & 0.816 & 2.000 & تراعي إدارة الكلية الثقافة التنظيمية في عملية & 7 \\
\hline 4 & 0.806 & 2.125 & تركز الخطة الإستراتيجية في الكلية علي رفع مستوي & 8 \\
\hline 1 & 0.447 & 2.750 & يلتزم العاملون في الكلية بقرارات الإدارة العليا & 9 \\
\hline 2 & 0.856 & 2.250 & تنمي إدارة الكلية الشعور بالانتماء للعمل في الكلية & 10 \\
\hline
\end{tabular}


من الجدول السابق يتضح أن المجال الأول مستوى انتشار ثقافة التخطيط الاستراتيجي جاء بنسبة متوسطة، وهذه النتيجة تختلف عن دراسة (الدجني، 2006) والتي أظهرت أن وضوح مفهوم التخطيط الاستراتيجي لدى عينة الدراسة جاءت بدرجة مرتفعة. حيث أن الفقرة "9" ( يلتزم العاملون في الكلية بقرارات الإدارة العليا) تحصلت على الرتبة الأولى بنسبة 2.75. وأن الفقرة "10" ( تنمى إدارة الكلية الشعور بالانتماء للعمل في الكلية) تحصلت علي الرتبة التانية بنسبة 2.25. وأن الفقرة "2"( تربط إدارة الكلية سياستها الاستراتيجية بثقافة المجتمع الأصلي) تحصلت على الرتبة الثالثة بنسبة 2.18. وأن الفقرة"8"(تركز الخطة الإستراتيجية في الكلية على رفع مستوي الخدمات التي تقدمها للمستفيدين)تحصلت علي الرتبة الرابعة بنسبة 2.12. وأن الفقرة"7"(تراعي أدارة الكلية الثقافة التنظيمية في عملية التخطيط) تحصلت علي الرتبة

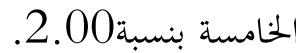

وأن الفقرتين"5,6"(يعمل العاملون ف الكلية بروح الفريق الواحد لإعداد الخطة المستقبلية) (تطلع إدارة الكلية العاملين فيها بإستمرار على خططها المستقبلية)تحصلتا على الرتبة السادسة بنسبة 1.93 إداه 1.93

وأن الفقرتين"1,3"(تشجع إدارة الكلية العاملين على المشاركةف التخطيط الاستراتيجي) (تحترم إدارة الكلية أراء العاملين فيها حول الخطة الاستراتيجية) تحصلتا على الرتبة السابعة بنسبة 1.87. وأن الفقرة "4"( تراعي إدارة الكلية الثقافة السائدة بين العاملين بما يخدم التخطيط الاستراتيجي) تحصلت على المرتبة الثامنة بنسبة 1.81. 


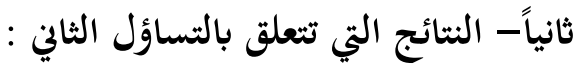

ينص هذا التساؤل على: ما مستوى ممارسة إدارة الكلية للتخطيط الاستراتيجي؟ ولإجابة على هذا التساؤل تم حساب المتوسطات الحسابية والانحراف المعياري

$$
\text { لاستجابات أفراد العينة، والجمدول رقم (2) يوضح ذلك: }
$$

جدول رقم (2) يبين المتوسطات الحسابية والانحرف المعياري لاستجابات أفراد العينة حول المجال الثاني

\begin{tabular}{|c|c|c|c|c|}
\hline 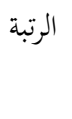 & المعياري & المتوسط & 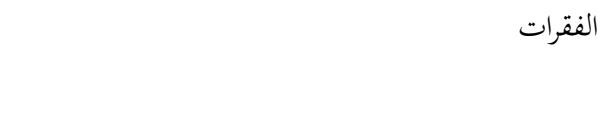 & 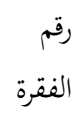 \\
\hline 7 & 0.806 & 1.875 & لدى إدارة الكلية خطة إستراتيجية على مستوى الأقسام & 1 \\
\hline 5 & 0.771 & 2.062 & تلتزم إدارة الكلية بالتخطيط المستمر لتطوير أدائها & 2 \\
\hline 7 & 0.885 & 1.875 & توفر إدارة الكلية الإمكانات التي من شأها تحقيق الخطة & 3 \\
\hline 4 & 0.718 & 2.125 & للتى إدارة الكلية المقدرة علي اتخاذ القرار السليم & 4 \\
\hline 2 & 1.032 & 2.500 & لدى إدارة الكلية رؤية واضحة للكلية & 5 \\
\hline 3 & 0.683 & 2.250 & تضع إدارة الكلية لنفسها غايات محددة & 6 \\
\hline 6 & 0.816 & 2.000 & تمتلك إدارة الكلية أهدافا طويلة الأجل & 7 \\
\hline 1 & 0.500 & 2.625 & لدى إدارة الكلية أهدافا قصيرة الأجل & 8 \\
\hline 5 & 0.771 & 2.062 & تشخص إدارة الكلية نقاط القوة والضعف في الكلية & 9 \\
\hline 7 & 0.619 & 1.875 & تلتزم إدارة الكلية بتنفيذ كل بنود الخطة الإستراتيجية & 10 \\
\hline
\end{tabular}

يتضح من الجدول السابق أن المجال الثاني مستوى محارسة التخطيط الاستراتيجي جاء بنسبة متوسطة، وهذه النتيجة تتفق مع دراسة (الأشقر، 2002). 
واقع التخطيط الاستراتيجي بكليات جامعة مصراتة

مجلة كلية الآداب- العدد خاص (2) الجلد (1)

عدد خاص بالورقات العلمية المقدمة للمؤثمر الدولي الثاني للتعليم في ليبيا، مصراتة، ليبيا، مارس 2019

حيث أن الفقرة"8" (لدي إدارة الكلية أهداف قصيرة الأجل) تحصلت على الرتبة الأولى

بنسبة 2.62.

وأن الفقرة "5" (لدي إدارة الكلية رؤية واضحة للكلية) تحصلت علي الرتبة الثانية بنسبة .2 .50

وأن الفقرة"6" (تضع إدارة الكلية لنفسها غايات محددة) تحصلت علي الرتبة الثالثة بنسبة .2 .25

وأن الفقرة"4" (لدي إدارة الكلية المقدرة على اتخاذ القرار السليم للتخطيط الاستراتيجي) تحصلت على الرتبة الرابعة بنسبة 2.12.

وأن الفقرتين "2،9" ( تلتزم إدارة الكلية بالتخطيط المستمر لتطوير أداهما ) (تشخص إدارة الكلية نقاط القوة والضعف في الكلية) تحصلتا علي الرتبة الخامسة بنسبة 2.06. وأن الفقرة "7" ( تمتلك إدارة الكلية أهدافاً طويلة الأجل ) تحصلت علي الرتبة السادسة بنسبة 2.00.

وأن الفقرات"1،10،3" (لدى إدارة الكلية خطة إستراتيجية علي مستوى الأقسام ) (توفر إدارة الكلية الإمكاناتالتي من شأها تحقيق الخطة الإستراتيجية ) (تلتزم إدارة الكلية بتنفيذ كل بنود الخطة الإستراتيجية) تحصلت علي الرتبة السابعة بنسبة 1.87.

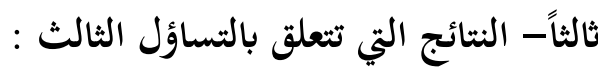

ينص هذا التساؤل على: ما مستوى كفاءة الموارد البشرية في عمليةالتخطيط الاستراتيجي؟ ولإجابة على هذا التساؤل تم حساب المتوسطات الحسابية والانحراف المعياري لاستجابات أفراد العينة، والجدول رقم (3) يوضح ذلك: 
واقع التخطيط الاستراتيجي بكليات جامعة مصراتة

مجلة كلية الآداب- العدد خاص (2) الجلد (1)

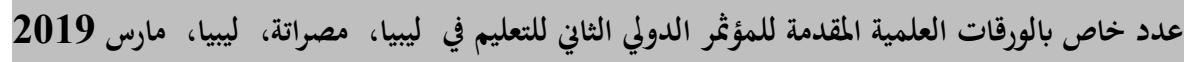

جلدول رقم (3) بيبن المتوسطات الحسابية والانحراف المعياري لاستجابات أفراد العينة حول المجال الثالث

\begin{tabular}{|c|c|c|c|c|}
\hline الرتبة & الانحراف & المتوسط & الفقرات & رقب \\
\hline 5 & 0.629 & 1.437 & لدى العاملين في الكلية معرفة بالتخطيط الاستراتيجي & 1 \\
\hline 1 & 0.853 & 1.937 & يتوافر لدى العاملين في الكلية القدرة على المشاركة في التخطيط & 2 \\
\hline 2 & 0.750 & 1.812 & توفر إدارة الكلية فرص المشاركة في المؤتمرات المحلية والعربية في بحال & 3 \\
\hline 2 & 0.834 & 1.812 & يمتلك العاملون الخبرة التي تمكنهم من إبداء رأيهم في تعديل الخطة & 4 \\
\hline 1 & 0.853 & 1.937 & يسعى العاملون على تطوير قدراقم وتحسينها وتنميتها في بجال & 5 \\
\hline 3 & 0.727 & 1.562 & تقع مسؤولية التخطيط الاستراتيجي على عاتق العاملين في الكلية & 6 \\
\hline 4 & 0.730 & 1.500 & تقدم إدارة الكلية التدريب الكافي للعاملين في مجال التخطيط & 7 \\
\hline
\end{tabular}

يتضح من الجمدول السابق أن المجال الثالث مستوى كفاءة الموارد البشرية جاء بنسبة

متوسطة. حيث أن الفقرتين "2،" ( لدى العاملين في الكلية معرفة بالتخطيط الاستراتيجي ) (يسعي العاملون على تطوير قدراتم وتحسينها وتنميتها في ججال التخطيط الاستراتيجي ) تحصلتا على الرتبة الأولى بنسبة 1.95. وأن الفقرتين "3،3" ( توفر إدارة الكلية فرص المشاركة في المؤتمرات المحلية والعربية في بجال التخطيط الاستراتيجي ) (يمتلك العاملون الخبرة التي تمكنهم من إبداء رأيهم فن تعديل الخطة الإستراتيجية) تحصلتا على الرتبة الثانية بنسبة 1.56. وأن الفقرة "6" ( تقع مسؤولية التخطيط الاستراتيجي على عاتق العاملين في الكلية)تحصلت على الرتبة الثالثة بنسبة 1.50. 
وأن الفقرة "7" ( تقدم إدارة الكلية التدريب الكافي للعاملين في مجال التخطيط الاستراتيجي) تحصلت على الرتبة الرابعة بنسبة 1.50.

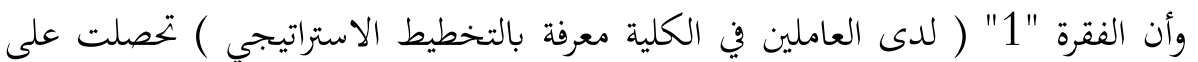
الرتبة الخامسة بنسبة 1.43.

\section{رابعاً- النتائج التي تنعلق بالتساؤل الرابع :}

ينص هذا التساؤل على: ما مدى توافر الموارد المالية في الكلية لخدمة عملية التخطيط الاستراتيجي؟ ولإججابة على هذا التساؤل تم حساب المتوسطات الحسابية والانحراف

$$
\text { المعياري لاستجابات أفراد العينة والجدول رقم (4) يوضح ذلك: }
$$

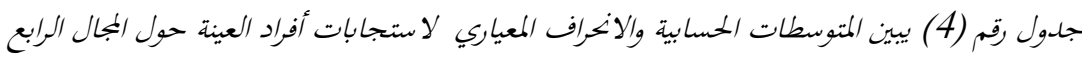

\begin{tabular}{|c|c|c|c|c|}
\hline الرتبة & الانحراف & المتوسط المسابي & الفقرات & رقم الفقرة \\
\hline 5 & 0.629 & 1.437 & تخصص إدارة الكلية جزءا مناسبا من موازنة & 1 \\
\hline 6 & 0.602 & 1.312 & 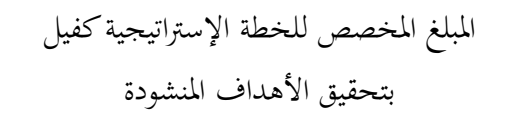 & 2 \\
\hline 3 & 0.500 & 1.625 & تعتمد أدارة الكلية في تنفيذ الخطة الإستراتيجية & 3 \\
\hline 5 & 0.512 & 1.437 & تعتمد أدراه الكلية علي إيرادات الطلاب في بناء & 4 \\
\hline 1 & 0.813 & 2.437 & ترتبط عملية التخطيط الاستراتيجي بمدي توفر & 5 \\
\hline 4 & 0.512 & 1.562 & تكفي الموارد المالية مراحل الخطة الإستراتيجية & 6 \\
\hline 2 & 0.655 & 1.812 & تشارك مؤسسات محلية في دعم عملية التخطيط & 7 \\
\hline
\end{tabular}




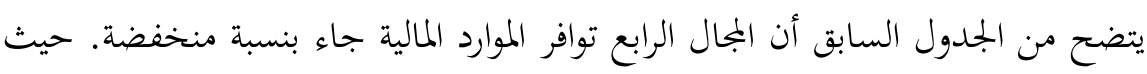

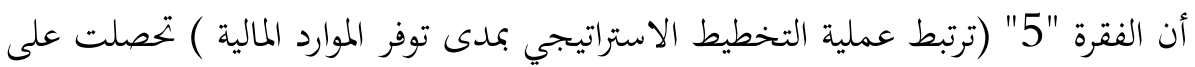
الرتبة الأولى بنسبة 2.43. وأن الفقرة "7" ( تشارك مؤسسات محلية في دعم عملية التخطيط الاستراتيجي) تحصلت على الرتبة الثانية بنسبة 1.81. وأن الفقرة "3" ( تعتمد إدارة الكلية في تنفيذ الخطة الإستراتيجية على المنح الخارجية) تحصلت الرتبة الثالثة بنسبة 1.62. وأن الفقرة "6" ( تكفى الموارد المالية مراحل الخطة الإستراتيجية ومستوياتا كافة ) تحصلت على الرتبة الرابعة بنسبة 1.56. وأن الفقرتين "4،1،" ( تخصص إدارة الكلية جزءاً مناسباً من موازنة الكلية للتخطيط الاستراتيجي) (تعتمد إدارة الكلية على إيرادات الطلاب في بناء خطتها الإستراتيجية) تحصلتا على الرتبة الخامسة بنسبة 1.43. وأن الفقرة"2" ( المبلغ المخصص للخطة الإستراتيجية كفيل بتحقيق الأهداف المنشودة ) تحصلت على الرتبة السادسة بنسبة 1.31. خامساً- النتائج التي تتعلق بالتساؤل الخامس : ينص التساؤل على: هل هناك فروق ذات دلالة إحصائية في استجابات أفراد العينة حول واقع التخطيط الاستراتيجي وفقاً لمتغير الجنس؟ ولإججابة على هذا التساؤل تم استخدام

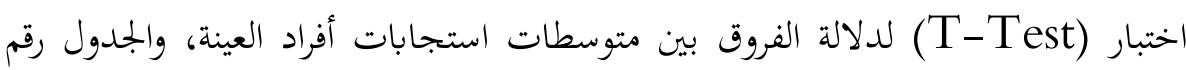
(5) - (5) يوضح ذلك: 
واقع التخطيط الاستراتيجي بكليات جامعة مصراتة

مجلة كلية الآداب- العدد خاص (2) الجلد (1)

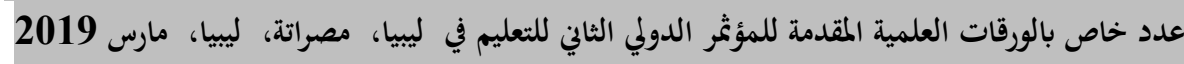

جدول رقم (5) يوضح اختبار (T-Test) لدلالة الفروق بين متوسطات استجابات أفراد العينة وفقاً لمتغير

الجنس

\begin{tabular}{|c|c|c|c|c|c|c|c|}
\hline الدلالة & الدستوى & درجة الحرية & قيمة ت & الالمعراف & المتوسط الحسابي & العدد & الجنس \\
\hline \multirow{2}{*}{ غالة } & \multirow[t]{2}{*}{0.082} & \multirow[t]{2}{*}{14} & \multirow[t]{2}{*}{1.874} & 13.625 & 72.7500 & 8 & ذكر \\
\hline & & & & 16.639 & 58.5000 & 8 & أنثى \\
\hline
\end{tabular}

من الجدول السابق يتضح أنه لا توجد فروق ذات دلالة إحصائية وفقاً لمتغير الجنس بين استجابات أفراد العينة، وهذه النتيجة تتفق مع دراسة (الشويخ، 2007)

ويرجع السبب في أنه لا توجد فروق في المهام والواجبات التي يؤديها كليهما سواء أكان

رئيس القسم ذكراً أم أنثى، وأن التخطيط الاستراتيجي لا يضع النوع عائقاً أمام القيام به.

\section{سادساً- النتائج التي تتعلق بالتساؤل السادس :}

ينص التساؤل على: هل هناك فروق ذات دلالة إحصائية في استجابات أفراد العينة حول

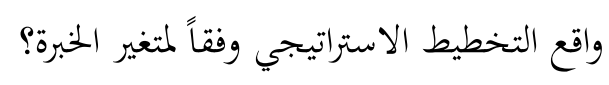

ولإججابة على هذا التساؤل تم استخدام التحليل التباين الأحادي لاستجابات أفراد العينة،

$$
\text { والجدول رقم (6) يوضح ذلك: }
$$

\begin{tabular}{|c|c|c|c|c|c|c|}
\hline الدلالة & مستوى الدلالة & قيمة F & متوسط & درجة الحرية & بموع المربعات & مصدر التباين \\
\hline \multirow{3}{*}{ غير دالة } & \multirow{3}{*}{0.112} & \multirow{3}{*}{2.603} & 579.058 & 2 & 1158.117 & بين المجموعات \\
\hline & & & 222.433 & 13 & 2891.633 & داخل المجموعات \\
\hline & & & & 15 & 4049.750 & الكلي \\
\hline
\end{tabular}

جدول رقم (6) يوضح تحليل التباين الأحادي لاستجابات أفراد العينة وفقاً لمتغير سنوات الخبرة 
من الجدول السابق يتضح أنه لا توجد فروق ذات دلالة إحصائية وفقاً لمتغير سنوات الخبرة بين استجابات أفراد العينة، وهذه النتيجة تتفق مع دراسة (الأشقر، 2002) وتختلف مع دراسة (الشويخ، 2007). وهذا يدل على أن سنوات الخبرة لرؤساء الأقسام

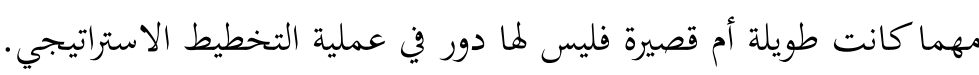

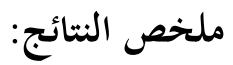
وبعد جمع الاستبانات تمت معالجة البيانات إحصائياً باستخدام برنامج الحزم الإحصائية للعلوم الاجتماعية (SPSS) وكانت النتائج على النحو الآتي: - أن مستوى انتشار ثقافة التخطيط الاستراتيجي لدى أفراد العينة جاء بنسبه متوسطة. - أن مستوى ممارسة التخطيط الاستراتيجي لدأفراد العينة جاء بنسبه متوسطة. - أن مستوى كفاءة الموارد البشرية لدى أفراد العينة جاء بنسبه متوسطة.

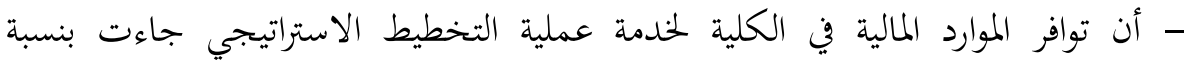
منخفضة. - عدم وجود فروق ذات دلالة إحصائية في استجابات أفراد العينة حول واقع التخطيط الاستراتيجي وفقا لمتغيرات الجنس، سنوات الخبرة. - التوصيات: وفي ضوء النتائج أوصت الباحتثان بعدة توصيات أهمها: 1- الاهتمام بعقد دورات تدريبية متخصصة بالمهارات الادراية عامة وبالتخطيط الاستراتيجي خاصةً. 2- ترسيخ ثقافة التخطيط الاستراتيجي داخل الكليات. 3- ضرورة توفير الموارد المالية لعملية التخطيط الاستراتيجي والتي من شأها أن تسهل من تصني تطوير الخطط والاستراتيجيات، وتحفز العاملين على العطاء والتقدم في بناء نظام تعليمي متقدم. 4- تذليل المشكلات والمعوقات التي من شأها أن تعين على عملية التخطيط الاستراتيجي. 
- المقترحات:وبناءً على النتائج وفي ضوء التوصيات تقترح الباحثتان الآتي: 1-إجراء دراسة مماثلة لواقع التخطيط الاستراتيجي بكليات أخرى بجامعة مصراتة ومقارنتها بالدراسة الحالية. 2-إجراء دراسة لأثر التدريب الخاصة بالتخطيط الاستراتيجي على مستوى ممارسته.

\section{قائمة المراجع:}

أبو شناق، نجاة أحمد محمد (2011).التخطيط الاستراتيجي للتدريب وأثثه على أداء العاملين(رسالة ماجستير غير منشورة) أكاديمية الدراسات العليا، طرابلس - ليبيا. الأشقر، إبراهيم يوسف (2002). واقع التخطيط الاستراتيجي للى مديري المنظمات

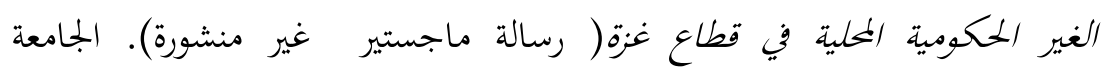

$$
\text { الإسلامية، غزة. }
$$

حافظ، صبري؛ البحيري، السيد (2006). تخطيط المؤسسات التعليمية، القاهرة: عالم

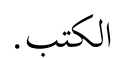

خطاب، عايدة سيد (1985).الإدارة والتخطيط الاستراتيجي في قطاع الأعمال والخدمات "سياسات إدارية" ، القاهرة: دار الفكر العربي.

خطاب، عايدة سيد (2003) .الإدارة الإستراتيجية للموارد البشرية في ظل إعادة الهيكلية الاندماج،مشاركة مخاطر، القاهرة: دار الحريري للطباعة.

درويش، عبد الكريم؛ تكلا، ليلى (1980) ـ أصول الإدارة العامة، القاهرة: مكتبة الانجلو المصرية.

الدجنى، إياد علي (2006). واقع التخطيط الاستراتيجي في الجامعة الإسلامية في ضوء

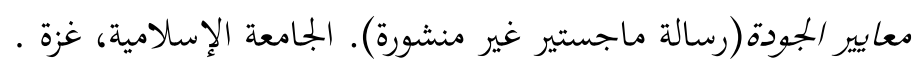
السلمي، علي (2000). الإدارة المعاصرة،مصر: مكتب الغريب. 
واقع التخطيط الاستراتيجي بكليات جامعة مصراتة

مجلة كلية الآداب- العدد خاص (2) الجلد (1)

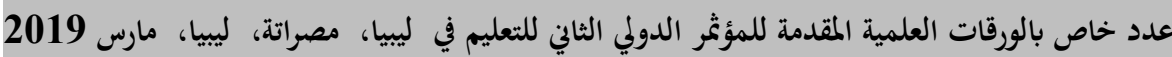

الشويخ، عاطف عبد الحميد (2007).واقع التخطيط الاستراتيجي في مؤسسات التعليم التقني في محافظات غزة(رسالة ماجستير غير منشورة). الجامعة الإسلامية،غزة

الشيخي، أم الخير عيسي (2008).العلاقة بين وضوح المفهوم العلمي للتخطيط الاستراتيجي ومستوى ممارسته في المشروعات الصناعية الصغيرة والمتوسطة (رسالة

$$
\text { ماجستير غير منشورة) أكاديمية الدراسات العليا - بنغازي }
$$

غنيم،محمد (2005).التخطيط التربوي،عمان: دار المسيرة للنشر والتوزيع. نور الدين، مازن سليم (2008).دور التخطيط الاستراتيجي في زيادة فاعلية الإدارة الملسرسية بمحافظات غزة (رسالة ماجستير غير منشورة). الجامعة الإسلامية، غزة. يونس، نزيه حسن حسين (2009).توظيف التخطيط الاستراتيجي في تطوير الأشراف التربوي في محافظات غزة(رسالة ماجستير غير منشورة). جامعة الإسالامية ، غزة. 